\title{
THE FREE GENUS OF DOUBLED KNOTS
}

\author{
CHARLES LIVINGSTON
}

(Communicated by Haynes R. Miller)

\begin{abstract}
The free genus of an untwisted doubled knot in $S^{3}$ can be arbitrarily large.
\end{abstract}

Every knot $K$ in $S^{3}$ bounds a surface $F$ for which $S^{3}-F$ is a solid handlebody. Such a surface is called a free Seifert surface and the minimal genus of such a surface is called the free genus of the knot, denoted $g_{f}(K)$. This note presents a proof of a statement made in Problem 1.20 of $[\mathbf{K}]$ : the free genus of an untwisted double of a knot in $S^{3}$ can be arbitrarily large. Problem $1.20 \mathrm{a}$ asks for relations between the free genus of a knot and its other invariants. Since an untwisted double of a knot has trivial Alexander polynomial, classical abelian invariants will provide no upper bounds on the free genus of a knot.

Yoav Moriah [Mo] has proved a similar result for twisted doubles. In essence he used dihedral representations of the knot group to study the free genus. These representations do not exist for untwisted doubles. Symmetric representations will be used here. Connectivity arguments permit us to avoid detailed calculations of homology groups. The use of irregular covers instead of regular ones will decrease the complexity of the covers involved.

A reference for the techniques of knot theory used here is $[\mathbf{R}]$. The covering space theory is contained in [Ma], and is summarized at the end of this paper. Homology groups are always with integer coefficients.

Notation. Let $J$ be the knot in $S^{1} \times B^{2}$ illustrated in Figure 1. If $h$ is an embedding of $S^{1} \times B^{2}$ into $S^{3}$ carrying $S^{1} \times\{0\}$ to a knot $K$, then $h(J)$ is called a double of $K$. It is called an untwisted double if $h(l)$ is null homologous in $S^{3}-K$, where $l$ is a longitude of $S^{1} \times B^{2}$. The untwisted double of $K$ will be denoted $D(K)$.

$K_{m}$ will denote the connected sum of $m$ copies of the $(2,5)$-torus knot. Figures 2 and 3 illustrate $K_{1}$ and $K_{3}$ respectively.

The rest of this paper is devoted to proving the inequality $g_{f}\left(D\left(K_{3 n+1}\right)\right) \geq 3 n / 5$.

ALGEBRAIC PRELIMINARIES. It will be shown that $S^{3}-D\left(K_{3 n+1}\right)$ has a connected 5-fold covering space, $M_{3 n+1}$, for which $\operatorname{rank}\left(H_{1}\left(M_{3 n+1}\right)\right) \geq 6 n+1$. The next proposition, similar to one in [Mo], then gives the desired inequality.

PROPOSITION. If $M$ is a connected $d$-fold cover of $S^{3}-K$ then $\operatorname{rank}\left(H_{1}(M)\right) \leq$ $2 d g_{f}(K)+1$.

PrOOF. By Van Kampen's Theorem $\operatorname{rank}\left(\pi_{1}\left(S^{3}-K\right)\right) \leq 2 g_{f}(K)+1 .\left(S^{3}-K\right.$ is built from the complement a Seifert surface for $K$ by adding a single 1-handle

Received by the editors March 20, 1987 and, in revised form, September 1, 1987.

1980 Mathematics Subject Classification (1985 Revision). Primary 57M25.

Supported in part by a grant from the NSF. 


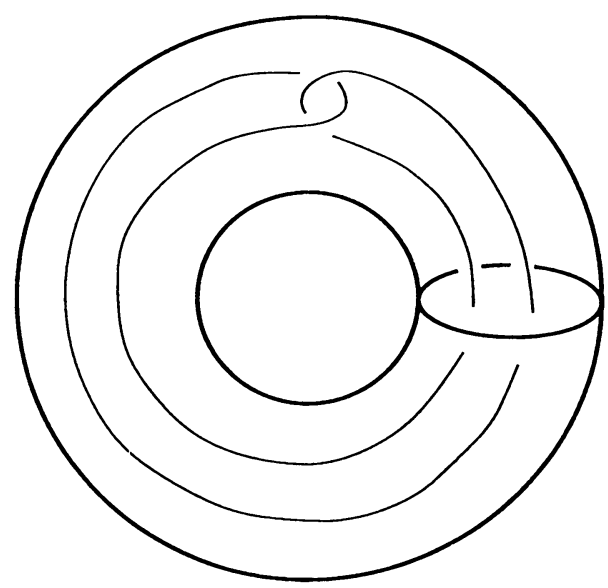

FIGURE 1

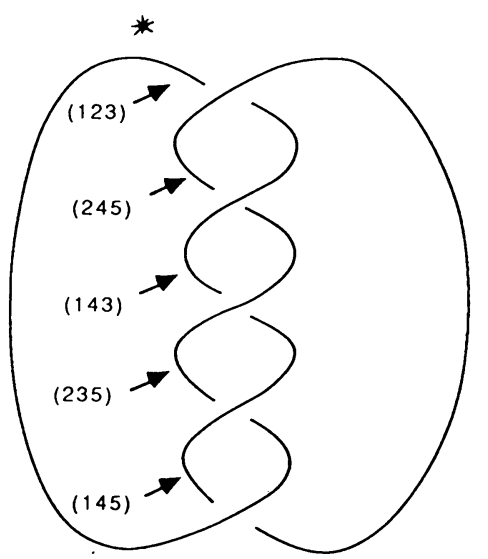

FIGURE 2

and 2-handles. The complement of a free Seifert surface of genus $g_{f}$ is a solid handlebody with $2 g_{f}$ handles.) The fundamental group of $M$ is isomorphic to an index $d$ subgroup of $\pi_{1}\left(S^{3}-K\right)$ and hence has rank at most $2 d g_{f}(K)+1$. This estimate follows from the fact that an index $d$ subgroup of a free group of rank $f$ is of $\operatorname{rank} d(f-1)+1$ [Ma, p. 204]. Finally, $\operatorname{rank}\left(H_{1}(M)\right) \leq \operatorname{rank}\left(\pi_{1}(M)\right)$.

The rest of the argument has three steps. In the first a transitive representation from $\pi_{1}\left(S^{3}-D\left(K_{3 n+1}\right)\right)$ to the fifth symmetric group, $S_{5}$, is constructed. This corresponds to a connected 5-fold cover of $S^{3}-D\left(K_{3 n+1}\right), M_{3 n+1}$. The next step, preliminary to estimating the homology of $M_{3 n+1}$, describes a geometric decomposition of $S^{3}-D\left(K_{m}\right)$. In the final step that decomposition is lifted to a decomposition of $M_{3 n+1}$ which quickly yields a bound on the rank of the homology.

1. The symmetric representation. Figure 2 illustrates the $(2,5)$-torus knot. A basepoint for the fundamental group of its complement is also marked. The labelling of the diagram specifies a representation of the fundamental group to $S_{5}$ using the following convention, described fully in [R]. $\pi_{1}\left(S^{3}-K\right)$ has a presentation 


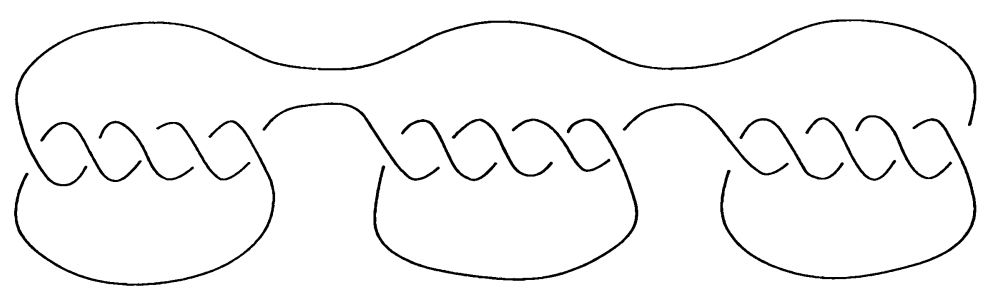

FIGURE 3

with one generator for each arc in the knot diagram. An arc corresponds to the element of $\pi_{1}$ represented by the path which runs from the basepoint to the arc, running above the diagram everywhere, goes once around the arc, and then returns to the basepoint. The label on each arc specifies the value of the representation on that element. The arrow indicates the direction in which the path goes around the arc. The representation is well defined if certain "Wirtinger" relations are satisfied. The details of these relations are described in $[\mathbf{R}]$. Checking them is an easy exercise for this example.

Note that in the above representation both the meridian and longitude to the knot represent to the 3-cycle $(123)$.

The connected sum of $(2,5)$-torus knots, as illustrated in Figure 3, has a similar representation. Construct such a representation by labelling the diagram for the connected sum. Just repeat the labelling used in Figure 2 on each factor in the connected sum. For this representation the meridian represents (123). The longitude represents to $(123)^{m}$, where $m$ is the number of factors.

Figure 4 illustrates the knot $J$ in $S^{1} \times B^{2}$. Here $S^{1} \times B^{2}$ is viewed as the complement of an unknotted circle in $S^{3}$. The labelling in that figure determines a representation of $\pi_{1}\left(S^{1} \times B^{2}-J\right)$ to $S_{5}$. Note that for this representation both the meridian and longitude of $S^{1} \times B^{2}$ represent to the 3-cycle (123).

The complement of $D\left(K_{m}\right)$ can be described as the union of the complement of $K_{m}$ with $S^{1} \times B^{2}-J$ along a torus. (See the next section for details.) By Van Kampen's theorem the representations on each piece determine a representation on the union if they agree on the intersection. This is the case if $m$ is of the form $3 n+1$.

2. Decomposing the knot complement. By construction there is a torus in the complement of $D\left(K_{3 n+1}\right)$ which separates the space into two components, one with closure $S^{1} \times B^{2}-J$ and the other with closure $S^{3}-N\left(K_{3 n+1}\right)$, the complement of an open tubular neighborhood of $K_{3 n+1}$. (These closures are taken as subspaces in the knot complement and not in $S^{3}$.) By the construction of connected sums there are $3 n$ annuli in the complement of $N\left(K_{3 n+1}\right)$ which together separate that space into $3 n+1$ components, each of which is homeomorphic to $S^{3}-K_{1}$.

Let $Z$ be the connected 2-complex consisting of the union of the separating torus and the annuli. Let $W=S^{3}-D\left(K_{3 n+1}\right)$. Finally, let $N(Z)$ be an open regular neighborhood of $Z$. We have the decomposition $W=(W-N(Z)) \cup \operatorname{cl}(N(Z))$.

$W-N(Z)$ has $3 n+2$ components, each with a single torus as boundary. The representation constructed above restricts to a transitive representation on the fundamental group of each of these components. The restriction of the representation to the fundamental group of $Z$ maps onto a cyclic subgroup generated by a 3-cycle. 


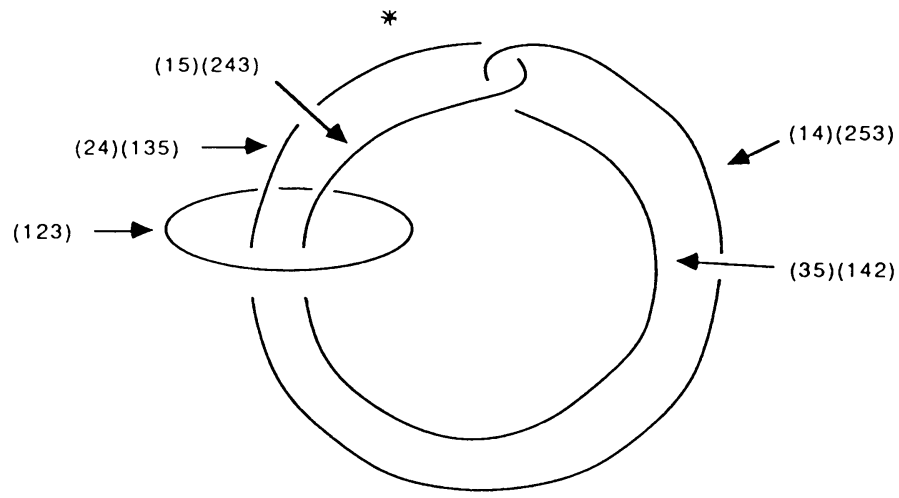

FIGURE 4

On each boundary component the representation also restricts to a map onto such a cyclic subgroup.

3. Decomposing the 5-fold cover. In this section we will let $X^{\prime}$ denote specified covering spaces of spaces $X$. The decomposition of $W$ just given lifts to a decomposition of the 5-fold cover of $W, M_{3 n+1}: M_{3 n+1}=(W-N(Z))^{\prime} \cup \operatorname{cl}(N(Z))^{\prime}$. $(W-N(Z))^{\prime}$ has $3 n+2$ components, each with 3 boundary components. $\operatorname{cl}(N(Z))^{\prime}$ has 3 components, with a total of $9 n+6$ boundary components. These calculations follow from covering space theory, contained in [Ma], and summarized below.

The rank of $H_{1}\left(M_{3 n+1}\right)$ can be estimated using the Mayer Vietoris sequence for this decomposition. $H_{1}\left(M_{3 n+1}\right)$ maps onto the kernel of the map of $H_{0}\left((W-N(Z))^{\prime} \cap \operatorname{cl}(N(Z))^{\prime}\right)$ to $H_{0}\left((W-N(Z))^{\prime}\right) \oplus H_{0}\left(\operatorname{cl}(N(Z))^{\prime}\right)$. This is the map of a free abelian group of rank $9 n+6$ to one of rank $3 n+5$. Hence the kernel has rank of at least $6 n+1$, as desired.

4. Covering space theory. Let $X$ be a space, $x$ a basepoint, and $\pi$ be its fundamental group. $G$ will denote a fixed group and $H$ a subgroup. Finally let $f$ be a homomorphism of $\pi$ to $G$. Under reasonable assumptions on the topology of $X$ there is a covering space of $X, X^{\prime}$, with the following properties. A bijection exists between the preimage of $x$ and the right cosets of $H$ in $G$. The group $\pi$ acts on the preimage of $x$ via lifting of paths and also acts on $G / H$ via $f$ and right multiplication. Under the mentioned bijection these two actions correspond. It can also be shown that the components of $X^{\prime}$ correspond to the orbits of $H$ under the right action.

The case used in this paper has $G=S_{5}$ and $H=S_{4} . \quad\left(S_{4}\right.$ is thought of as the stabilizer of $\{1\}$.) $G / H$ has 5 cosets. Hence representations to $S_{5}$ yield 5 -fold covers. If a representation of $\pi$ is transitive the action of $\pi$ on the cosets of $G / H$ is also transitive, so the cover is connected. If the representation of $\pi$ is onto a subgroup generated by a single element $g$ then one can check that there is one orbit corresponding to each cycle in a cyclic decomposition of $g$. We used the case of a 3-cycle, which has three cycles in its decomposition. $\left(\left(\begin{array}{lll}1 & 2 & 3\end{array}\right)=\left(\begin{array}{lll}1 & 23\end{array}\right)(4)(5).\right)$

REMARK. The argument above works for twisted doubles as well as untwisted ones. The only modification required is that a different number of factors is needed in the connected sum. 
ACKNOWLEDGMENT. Thanks are due to Richard Skora for his helpful comments regarding this work.

\section{REFERENCES}

[K] R. Kirby, Problems in low dimensional manifold theory, Proc. Sympos. Pure Math., vol. 32, Amer. Math. Soc., Providence, R. I., 1978, pp. 273-312.

[Ma] W. S. Massey, Algebraic topology: An introduction, Springer-Verlag, Berlin and New York, 1984.

[Mo] Y. Moriah, On the free genus of knots, Proc. Amer. Math. Soc. 99 (1987), 373-380.

[R] D. Rolfsen, Knots and links, Publish or Perish, Berkeley, Calif., 1976.

Department of MATHEMATICS, Indiana UNiversity, BloOMington, Indiana 47405 